\title{
Accelerated Fibrinolysis and Its Propagation on Vascular Endothelial Cells by Secreted and Retained tPA
}

\author{
Tetsumei Urano and Yuko Suzuki \\ Department of Medical Physiology, Hamamatsu University School of Medicine, 1-20-1 Handayama, Higashi-ku, \\ Hamamatsu 431-3192, Japan \\ Correspondence should be addressed to Tetsumei Urano, uranot@hama-med.ac.jp
}

Received 5 April 2012; Revised 11 July 2012; Accepted 20 July 2012

Academic Editor: Robert J. Parmer

Copyright ( $\odot 2012$ T. Urano and Y. Suzuki. This is an open access article distributed under the Creative Commons Attribution License, which permits unrestricted use, distribution, and reproduction in any medium, provided the original work is properly cited.

\begin{abstract}
We successfully visualized the secretory dynamics of tissue-type plasminogen activator (tPA) tagged by green fluorescent protein (tPA-GFP) from cultured vascular endothelial cells (VECs) using total internal reflection fluorescence (TIRF) microscopy and demonstrated that tPA-GFP secreted from VECs was retained on cell surfaces in a heavy-chain-dependent manner. Progressive binding of Alexa568-labeled Glu-plasminogen was also observed on the surface of active tPA-GFP expressing cells via lysine binding sites (LBS), which was not observed on inactive mutant tPA-GFP expressing cells. These results suggest that retained tPA on VECs effectively activated plasminogen to plasmin, which then facilitated the binding of additional plasminogen on the cell surface by proteolytically cleaving surface-associated proteins and exposing their C-terminal lysine residues. Thus prolonged retention of tPA appeared to play an important role in initiating and amplifying plasmin generation on VECs. LBS-dependent binding of plasminogen was also observed as a narrow band at the lytic front of the fibrin mesh formed on active tPA-GFP expressing cells, which expanded outward as the lytic area increased. This binding was not observed on inactive mutant tPA-GFP expressing cells or in the presence of aprotinin. The binding of plasminogen to partially digested fibrin appears to be indispensable for spontaneous fibrinolysis.
\end{abstract}

\section{Introduction}

Fibrinolysis takes place when undesirable fibrin is formed or when a hemostatic thrombus becomes unnecessary. Plasminogen activation by plasminogen activators (PAs) is the initial step in fibrinolysis, which is regulated by many physiological and pathological factors [1] so as to quickly dissolve unnecessary thrombi. Tissue-type PA (tPA) and urokinase-type PA (uPA) are the physiological PAs, and the former is synthesized and released from vascular endothelial cells (VECs) as an active form to initiate intravascular thrombolysis. Its characteristic of binding to fibrin is believed essential in the effective activation of plasminogen on the fibrin surface as well as in plasmin-catalyzed fibrin digestion [2]. The binding of plasminogen to fibrin through lysinebinding sites (LBS) existing in kringle domains is also believed to play an essential role in its effective activation on the fibrin surface [1]. In this paper, we introduce our recent findings on tPA secretory dynamics from VECs, and on the subsequent process of secreted tPA catalyzing plasminogen activation and fibrinolysis on vascular endothelial cells. Indispensiveness of the binding of plasminogen to partially digested fibrin in spontaneous fibrinolysis is also demonstrated.

\section{Secretion of tPA from VECs and Regulation of Its Activity in Plasma}

tPA is synthesized and released from VECs as an active form, either constitutively or in a regulated fashion following various kinds of stimuli from several different granules [5], and thus the amount of secreted tPA is a principal determinant of plasminogen activation potential in the vasculature. Another determinant of this potential is plasminogen activator inhibitor type 1 (PAI-1), the primary inhibitor of both tPA and uPA under physiological conditions. Since PAI1 exists in plasma in molar excess over tPA, most of the tPA circulates in an inactive complexed form with PAI-1, and 

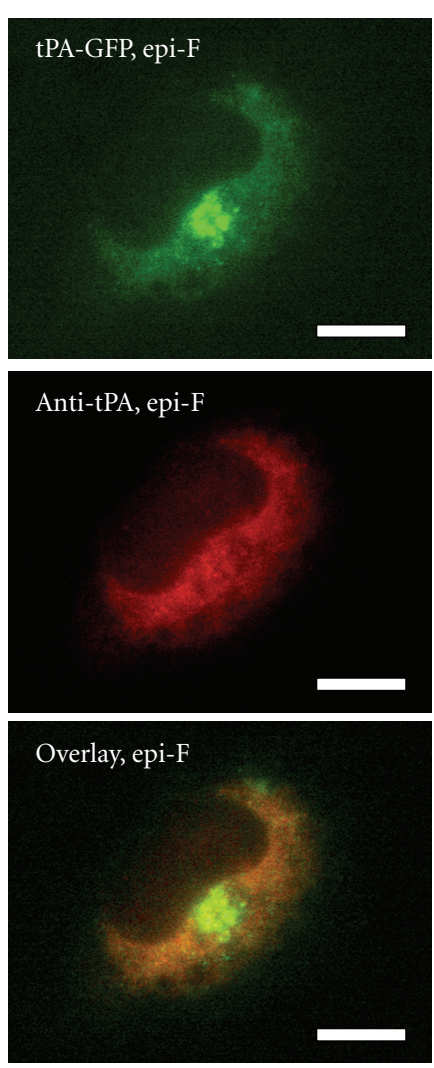

(a) Immunofluorescence image of tPA-GFP expressing EA hy926 cells
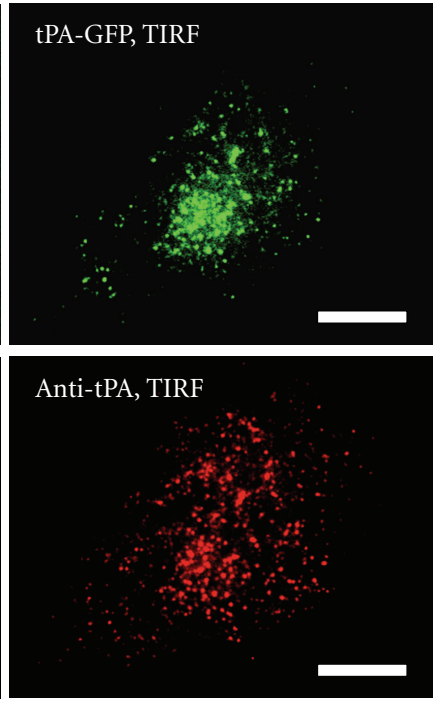

Overlay, TIRF

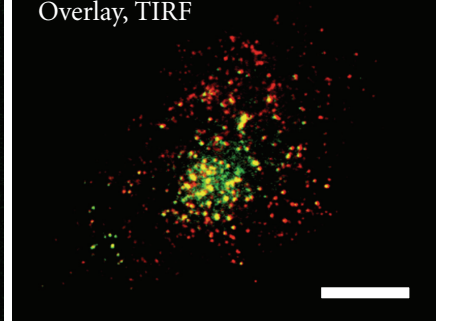

a-tPA

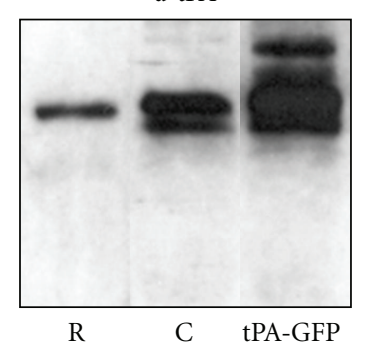

(b) Western blotting

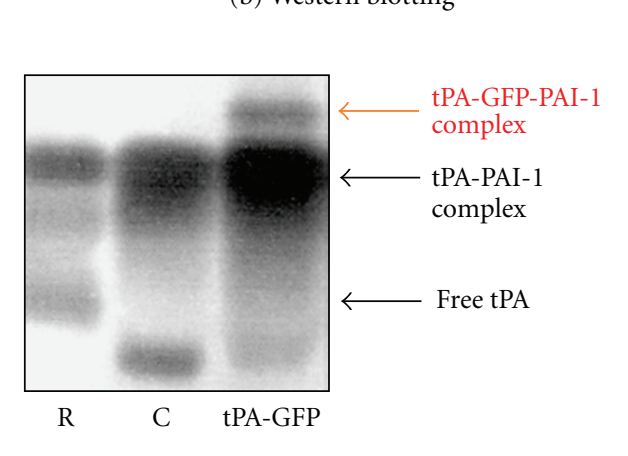

R: recombinant protein

C: control cells

(c) Fibrin autography

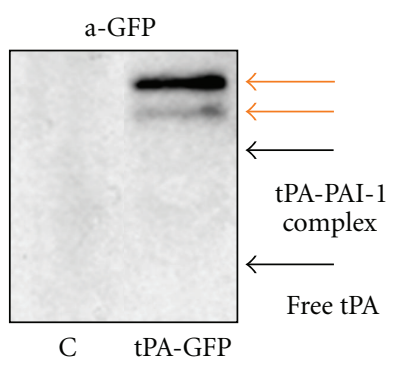

(1)

FIGURE 1: Characteristics of tPA-GFP expressing EA hy926 cells. (a) Immunofluorescence images of tPA-GFP expressing EA hy926 cells were captured by either epifluorescence microscopy (epi-F) or total internal reflection fluorescence microscopy (TIRF), the latter of which more clearly detects tPA-GFP containing granules as dots. (b) Culture media of tPA-GFP expressing EA hy926 cells and control cells were analyzed by western blotting. Additional high-molecular-weight bands detected by both anti-tPA and anti-GFP were observed in tPA-GFP expressing EA hy926 cells (orange arrows), suggesting that tPA-GFP has activity to form high-molecular-weight complex with PAI-1. (c) Fibrin autography of the same culture media. The additional bands detected by western blot analysis showed a lytic band (orange arrow). This figure was originally published in [3].

only a small fraction of this total tPA circulates as an active enzyme [6].

\section{3. tPA Secretory Dynamics}

3.1. Slow Kinetics of tPA Secretion. The human vascular endothelial cell line EA.hy926 was transfected with an expression vector encoding tPA tagged by green fluorescent protein (tPA-GFP), and tPA secretory dynamics were analyzed using total internal reflection fluorescence (TIRF) microscopy (Figure 1(a)) [3]. The expressed tPA-GFP was confirmed to be active by the facts that it formed a highmolecular-weight complex with PAI-1 (Figure 1(b)) and that it developed a lytic band on a plasminogen-rich fibrin overlay (Figure 1(c)). The sizes and distribution of tPA-GFP containing granules were indistinguishable from those of intrinsic wild tPA, which was detected using an anti-tPA antibody. TIRF microscopy was used to visualize tPA-GFP-containing granules, which were detected as dots when they appeared in the so-called evanescent field, which is approximately $100 \mathrm{~nm}$ from the interface. The secretory dynamics of docking and opening of tPA-GFP containing granules followed by
tPA-GFP secretion were appreciated by a sudden increase in fluorescent intensity followed by its gradual decrease. The most striking peculiarity in tPA secretory dynamics was that the release of tPA-GFP from the opened granule was very slow, which was shown by a slow decline in the fluorescent signal as well as by the persistence of an apparent fluorescent spot even 2-3 minutes after the opening of the granule. The mechanism for the long retention of tPA-GFP on membrane surfaces after granular opening was analyzed using several domain-deleted mutants of tPA-GFP. A mutant lacking the entire heavy chain and having only the catalytic domain disappeared quickly after granular opening, similar to the dynamics of a peptide hormone like insulin, suggesting that the prolonged retention of tPA requires the heavy chain.

\section{Retention of tPA on the Cell Surface after Exocytosis and Its Release by PAI-1}

Heavy-chain-dependent retention of tPA on the surface of VECs seems beneficial as it maintains an elevated plasminogen activation potential on the surface of the vasculature. The facts that in the culture medium of EA.hy926 cells tPA 

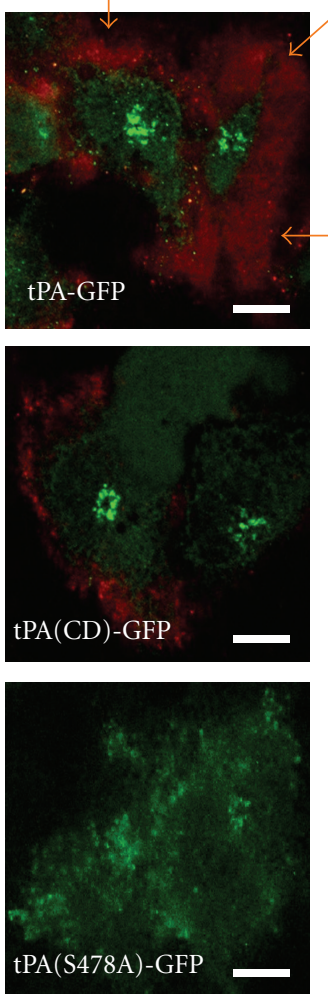

(a)

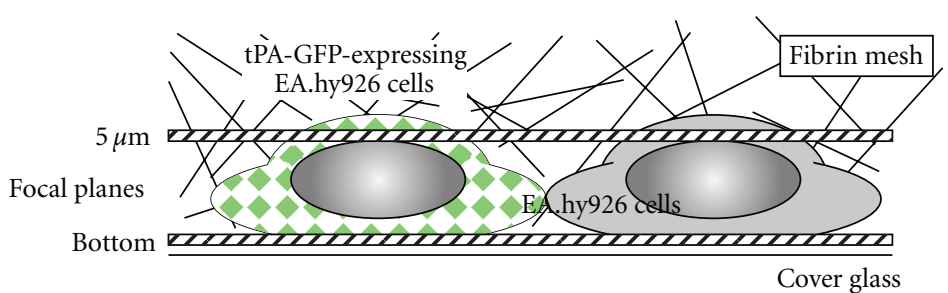

(b)

tPA-GFP, bottom, $10 \mathrm{~min}$

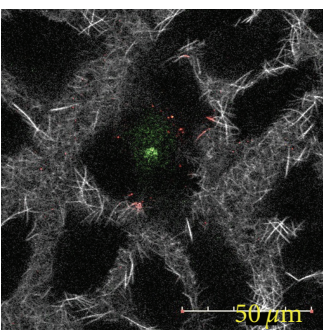

tPA-GFP, $5 \mu \mathrm{m}, 10 \mathrm{~min}$
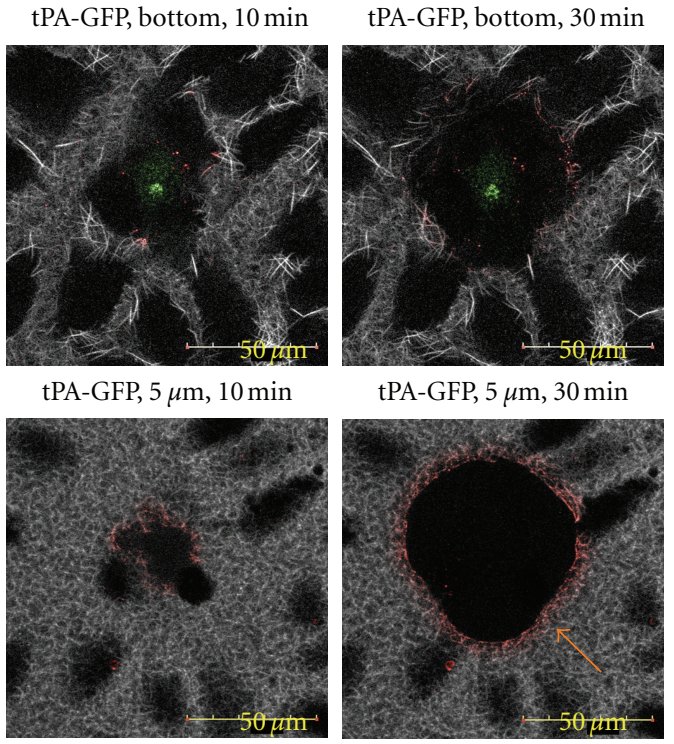

tPA-GFP, $5 \mu \mathrm{m}, 30 \mathrm{~min}$

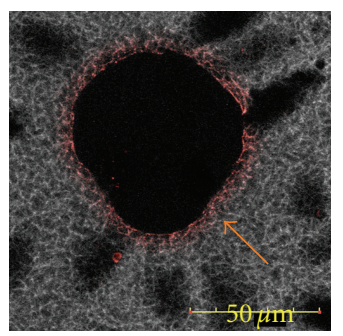

tPA-GFP, bottom, $50 \mathrm{~min}$

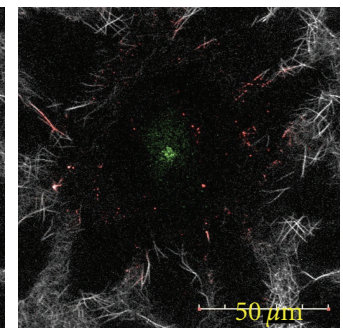

tPA-GFP, $5 \mu \mathrm{m}, 50 \mathrm{~min}$

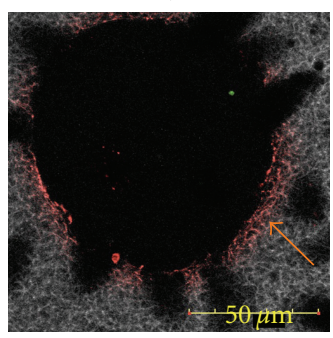

(c)

FIGURE 2: Accumulation of plasminogen and effective fibrinolysis on tPA-GFP-expressing EA hy926 cells. (a) Alexa568-labeled Gluplasminogen (568-Glu-plg) was incubated with EA.hy926 transfected with tPA-GFP, and the binding of plasminogen on the cell surface was observed (orange arrow), which was suppressed when either heavy-chain-deleted tPA-GFP (tPA(CD)-GFP) or catalytically inactive tPAGFP (tPA(S478A)-GFP) was employed. (b) A fibrin network was formed on EA.hy926 transfected with tPA-GFP using Alexa647-labeled fibrinogen, and its spontaneous lysis was monitored by confocal microscopy. (c) Fibrinolysis initiated by tPA-GFP expressing cells, and its gradual expansion was clearly observed both focal planes of the bottom and $5 \mu \mathrm{m}$ above the bottom. Linear binding of 568 -Glu-plg was also observed at the lytic front (orange arrow), which expanded outward as the lytic area increased. A part of this figure was originally published in $[4]$.

exists mostly as tPA-PAI- 1 complex and that free tPA rarely exists prompted us to analyze the potential effect of PAI1 on tPA retention. The following three results suggested that PAI-1 facilitated the release of tPA from the surface of VECs. First, following suppression of PAI-1 expression in EA.hy926 cells by siRNA, the retention time of tPA-GFP was prolonged. Second, supplementation of recombinant PAI1 , in turn, shortened the retention time and increased the amount of tPA-GFP-PAI-1 complex but not of free tPAGFP in the supernatant. Finally, a mutant tPA-GFP whose active site serine was substituted by alanine so as not to react with PAI-1 stayed on the cell surface for a longer time, and essentially no tPA-GFP was detected in the supernatant. Thus PAI-1 appeared to attenuate the fibrinolytic potential not only in plasma but also on the surface of VECs.

\section{Fibrinolysis Initiated by tPA Secreted from VECs}

tPA effectively activates plasminogen when it is bound to solid structures including fibrin [2], denatured proteins having a crossed beta-structure [7], and cell surfaces [2]. To achieve effective fibrinolysis, synchronized binding of plasminogen to these surfaces is necessary. When Alexa568labeled Glu-plasminogen (568-Glu-plg) was incubated with EA.hy926 transfected with tPA-GFP, progressive binding of plasminogen to the cell surface in an LBS-dependent manner was observed (Figure 2(a)) [4]. This accumulation of plasminogen on the cell surface was strongly attenuated when catalytically inactive mutant tPA-GFP was employed or when inhibitors of tPA or plasmin were supplemented, suggesting that plasmin-catalyzed exposure of new C-terminal lysine residues is involved in this binding process (Figure 3 ).

To analyze how surface-retained tPA on VECs effectively initiates fibrinolysis, a fibrin network was formed on EA.hy926 transfected with tPA-GFP using Alexa647-labeled fibrinogen (647-fbg) and its spontaneous lysis was monitored using confocal microscopy (Figure 2(b)) [4]. Monitoring at several sequential planes revealed that fibrinolysis began at the surface of tPA-GFP expressing cells and gradually expanded (Figure 2(c)). Importantly, linear binding of 568-Glu-plg was always observed at the lytic 


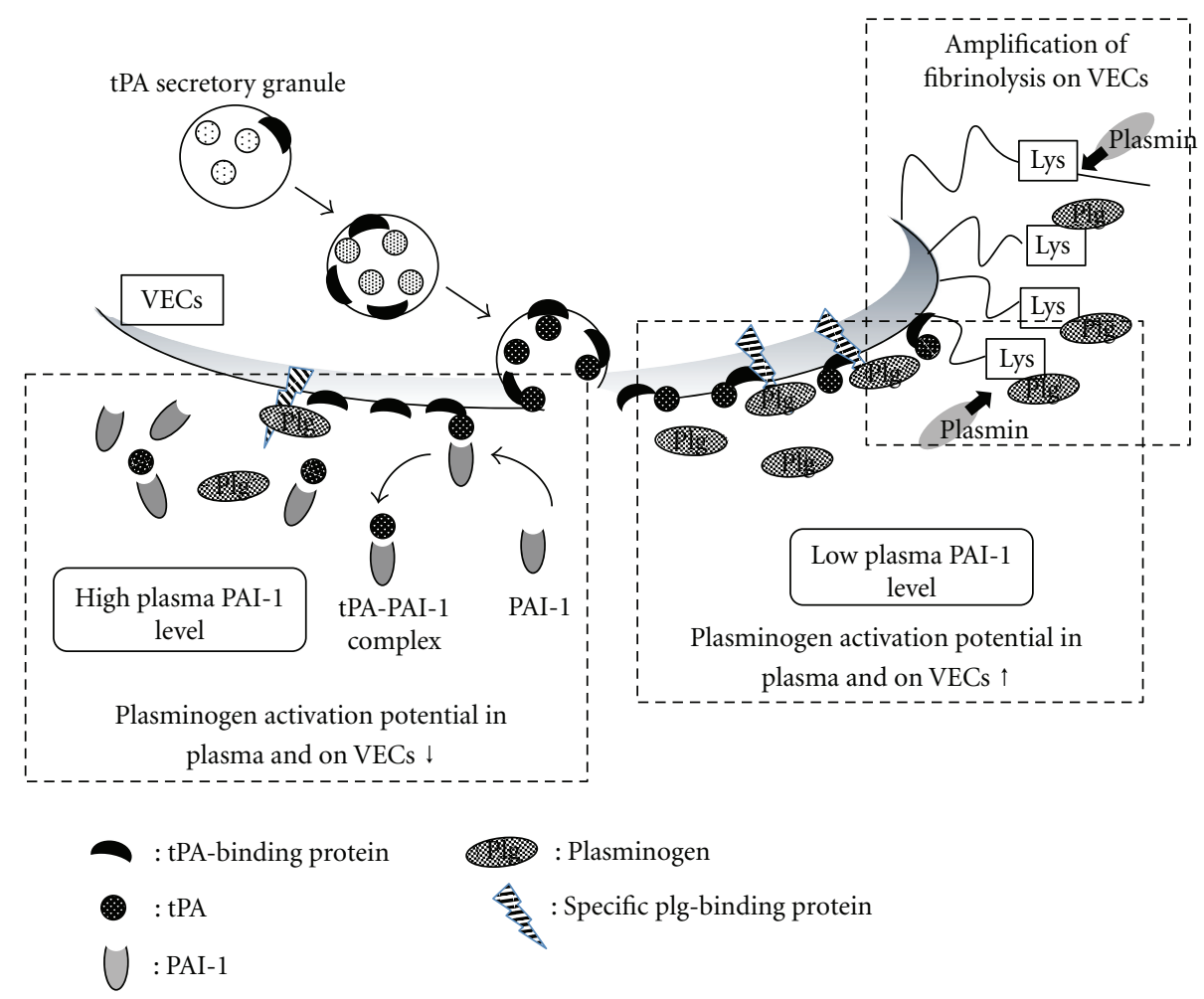

FIGURE 3: Mechanism to maintain a high fibrinolytic potential on VECs and its modification by PAI-1. Secreted- and retained-tPA readily activates cell-bound plasminogen on VECs (lower right), which amplifies plasminogen accumulation to newly exposed C-terminal lysine by plasmin-dependent cleavage of cell surface proteins (upper right). PAI-1 suppresses these mechanisms by removing retained tPA from VECs by forming high molecular weight complex (left).

front, which expanded outward as the lytic area increased. Presumably the 568-Glu-plg bound preferentially to newly exposed C-terminal lysine residues by plasmin-catalyzed partial digestion of fibrin fibers. As was shown before using many different methods [1] and was recently confirmed by analyzing the crystal structure of plasminogen [8], Gluplasminogen seems to have undergone a conformational change from its closed conformation to its easily activatable open form, and this likely facilitates clot lysis. Thus, as has been expected for a long time, the binding of Glu-plg to fibrin that has been partially cleaved by plasmin clearly proved essential for spontaneous initiation of clot lysis.

\section{Cell-Associated Fibrinolysis and Cell Function}

Plasminogen activation and the associated fibrinolysis on cell surfaces are known to play a variety of functions under both physiological and pathological conditions [2, $9,10]$. Plasminogen binding-molecules play central role in these events, and several candidate molecules have been reported such as Plg- $\mathrm{R}_{\mathrm{KT}}$, histone $\mathrm{H} 2 \mathrm{~B}$, and annexin A2S100A10 complex as well as alpha-enolase. The binding of plasminogen and/or its activation to plasmin on these molecules has been suggested to possibly transmit signals into the cells and to modify cellular functions $[11,12]$. These specific plasminogen binding molecules, therefore, seem to play important roles in the initiation of effective plasmin generation, as well as in cell signaling, both of which are important in modifying cellular functions. Our findings, however, that the accumulation of plasminogen increased during the time course of tPA secretion from VECs suggests that other proteins also function as plasminogen binding molecules on the cell surface by providing newly exposed $\mathrm{C}$-terminal lysine residues after plasmin-catalyzed cleavage (Figure 3). We believe that this augmentation of plasminogen binding by newly exposed C-terminal lysines as well as its effective activation also plays essential roles not only in the degradation of fibrin or matrix proteins, but also in proteasedependent signal transduction.

\section{Summary}

We have described a unique mechanism of tPA secretion and its role in effective fibrinolysis that takes place on VECs. This mechanism seems to require retention of tPA after secretion as well as the binding of plasminogen to specific molecules on the cell surface. Following the exposure of new Cterminal lysines on membrane proteins by plasmin-catalyzed cleavage, further accumulation of plasminogen on the cell surface was observed. Such initiation and augmentation of plasminogen activation seems to be important in modifying cell functions involved in a variety of physiological and pathological events. Understanding these mechanisms in greater detail will provide us with new strategies to control these events. 


\section{Acknowledgments}

The research summarized here was originally published in Blood [3, 4], () the American Society of Hematology. This work was supported by a Grant-in-Aid for Scientific Research (C: 19590858) to Y. Suzuki and (C: 24590273) to T. Urano from the Japan Society for the Promotion of Science (JSPS), Japan Heart Foundation Research Grant, a grant from the Inamori Foundation to Y. Suzuki, and grants from the Smoking Research Foundation and SENSHIN Medical Research Foundation to T. Urano.

\section{References}

[1] F. J. Castellino and V. A. Ploplis, "Structure and function of the plasminogen/plasmin system," Thrombosis and Haemostasis, vol. 93, no. 4, pp. 647-654, 2005.

[2] D. C. Rijken and H. R. Lijnen, "New insights into the molecular mechanisms of the fibrinolytic system," Journal of Thrombosis and Haemostasis, vol. 7, no. 1, pp. 4-13, 2009.

[3] Y. Suzuki, H. Mogami, H. Ihara, and T. Urano, "Unique secretory dynamics of tissue plasminogen activator and its modulation by plasminogen activator inhibitor- 1 in vascular endothelial cells," Blood, vol. 113, no. 2, pp. 470-478, 2009.

[4] Y. Suzuki, H. Yasui, T. Brzoska, H. Mogami, and T. Urano, "Surface-retained tPA is essential for effective fibrinolysis on vascular endothelial cells," Blood, vol. 118, no. 11, pp. 31823185, 2011.

[5] L. Knipe, A. Meli, L. Hewlett et al., "A revised model for the secretion of tPA and cytokines from cultured endothelial cells," Blood, vol. 116, no. 12, pp. 2183-2191, 2010.

[6] T. Urano, K. Sumiyoshi, M. H. Pietraszek, Y. Takada, and A. Takada, "PAI-1 play an important role in the expression of $t$ PA activity in the euglobulin clot lysis by controlling the concentration of free t-PA," Thrombosis and Haemostasis, vol. 66, no. 4, pp. 474-478, 1991.

[7] M. F. B. G. Gebbink, B. Bouma, C. Maas, and B. N. Bouma, "Physiological responses to protein aggregates: fibrinolysis, coagulation and inflammation (new roles for old factors)," FEBS Letters, vol. 583, no. 16, pp. 2691-2699, 2009.

[8] R. Law, T. Caradoc-Davies, N. Cowieson et al., "The X-ray crystal structure of full-length human plasminogen," Cell Reports, vol. 1, no. 3, pp. 185-190, 2012.

[9] R. Das, E. Pluskota, and E. F. Plow, "Plasminogen and its receptors as regulators of cardiovascular inflammatory responses," Trends in Cardiovascular Medicine, vol. 20, no. 4, pp. 120-124, 2010.

[10] E. C. Flood and K. A. Hajjar, "The annexin A2 system and vascular homeostasis," Vascular Pharmacology, vol. 54, no. 3-6, pp. 59-67, 2011.

[11] L. A. Miles, S. B. Hawley, N. Baik, N. M. Andronicos, F. J. Castellino, and R. J. Parmer, "Plasminogen receptors: the sine qua non of cell surface plasminogen activation," Frontiers in Bioscience, vol. 10, no. 2, pp. 1754-1762, 2005.

[12] B. Casar, Y. He, M. Iconomou, J. D. Hooper, J. P. Quigley, and E. I. Deryugina, "Blocking of CDCP1 cleavage in vivo prevents Akt-dependent survival and inhibits metastatic colonization through PARP1-mediated apoptosis of cancer cells," Oncogene, 2011. 

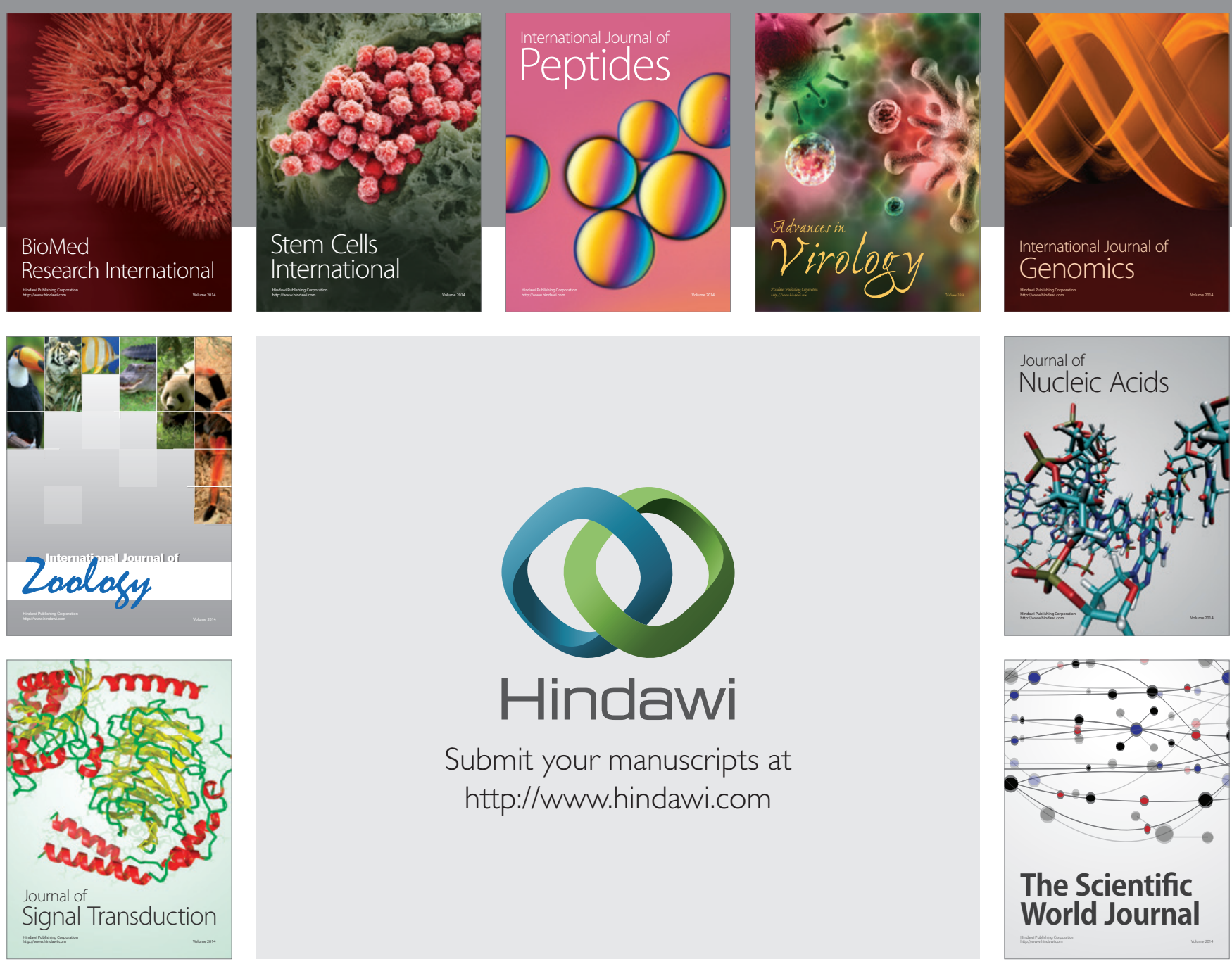

Submit your manuscripts at

http://www.hindawi.com
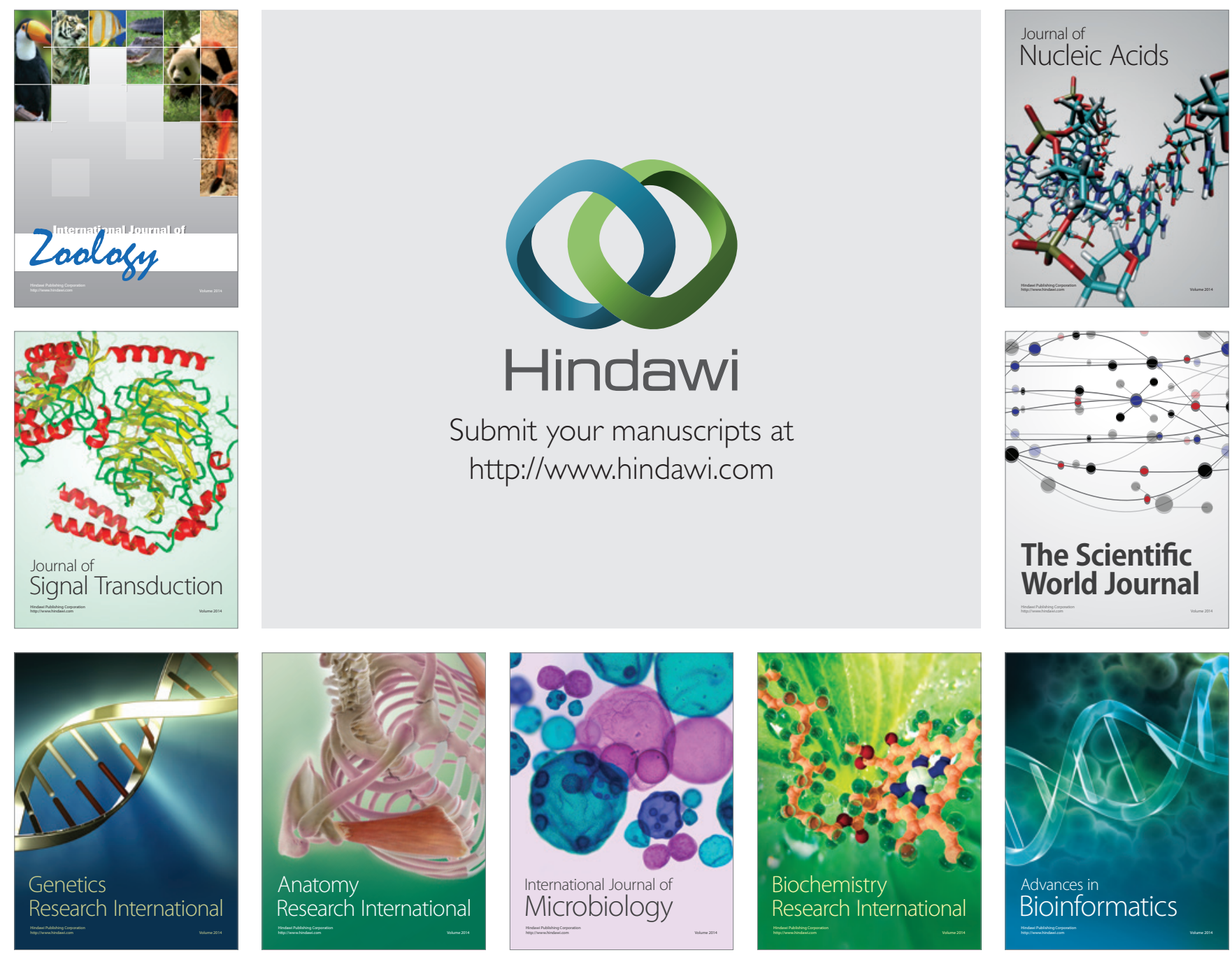

The Scientific World Journal
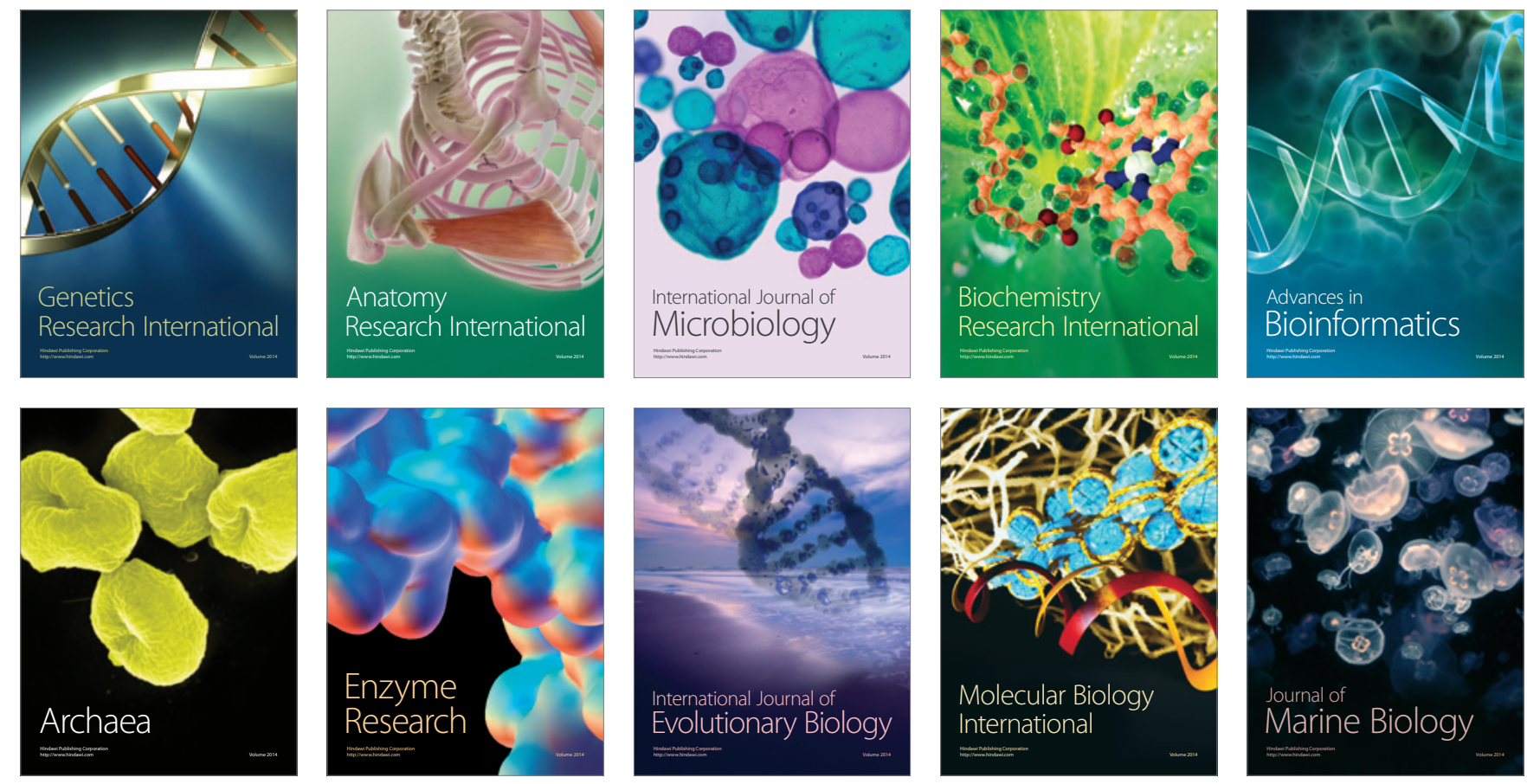